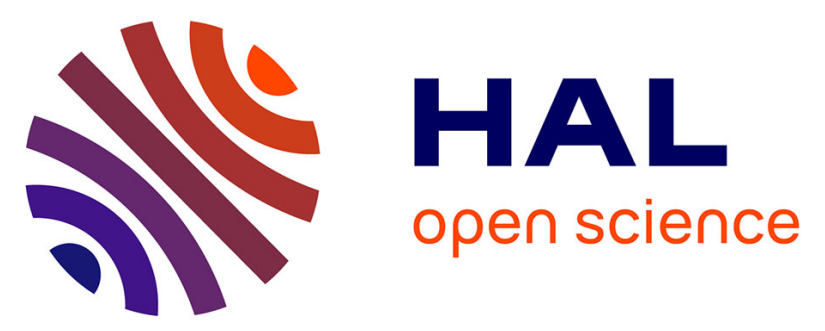

\title{
Interactions aérosols- rayonnement-climat en région méditerranéenne Impact de l'effet radiatif direct sur le cycle de l'eau
}

\author{
Marc Mallet, François Dulac, Pierre Nabat, Paola Formenti, Jean Sciare, \\ Gregory Roberts, Cyrielle Denjean, Jacques Pelon, Didier Tanré, Frédéric \\ Parol, et al.
}

\section{To cite this version:}

Marc Mallet, François Dulac, Pierre Nabat, Paola Formenti, Jean Sciare, et al.. Interactions aérosolsrayonnement-climat en région méditerranéenne Impact de l'effet radiatif direct sur le cycle de l'eau. La Météorologie, 2015, 91, pp.29-38. 10.4267/2042/57860 . insu-01233173

\section{HAL Id: insu-01233173 \\ https://hal-insu.archives-ouvertes.fr/insu-01233173}

Submitted on 12 Dec 2019

HAL is a multi-disciplinary open access archive for the deposit and dissemination of scientific research documents, whether they are published or not. The documents may come from teaching and research institutions in France or abroad, or from public or private research centers.
L'archive ouverte pluridisciplinaire HAL, est destinée au dépôt et à la diffusion de documents scientifiques de niveau recherche, publiés ou non, émanant des établissements d'enseignement et de recherche français ou étrangers, des laboratoires publics ou privés. 


\section{Résumé}

Une grande campagne expérimentale a été réalisée en Méditerranée occidentale pendant l'été 2013 afin d'étudier l'impact des aérosols sur le bilan radiatif et le climat régional. Les observations obtenues dans plusieurs stations de surface, à bord de deux avions de recherche, sous ballons sondes plafonnants et par satellite ont documenté la distribution et les propriétés physicochimiques et optiques des particules. Cette campagne a vu se succéder des transports d'aérosols désertiques d'intensité modérée, absorbant modérément le rayonnement solaire et stratifiés verticalement. Les premières simulations climatiques indiquent un impact significatif des aérosols notamment sur la température de surface de la mer, les flux océan-atmosphère et les précipitations saisonnières.

\section{Abstract}

Aerosol-radiation and climate interactions in the Mediterranean region. Impact of radiative effect on the water cycle

An experimental campaign, coupled with three-dimensional modeling, was conducted in the western Mediterranean during the summer of 2013 to study the impact of aerosols on the radiative balance and climate of this region. In situ observations were obtained on the ground, aboard two research aircraft and balloons to characterize the physico-chemical and optical properties of particles and their vertical stratification. This campaign was mainly characterized by moderate events of desert aerosols. During these episodes, strong vertical stratification was observed and the measurements of the optical properties reveal moderate absorbing particles in the visible spectrum. Climate simulations indicate a significant impact of aerosols in particular by changing the surface temperature of the sea, the ocean-atmosphere fluxes and consequently seasonal precipitation.

\title{
Interactions aérosols- rayonnement-climat en région méditerranéenne
}

\author{
Impact de l'effet radiatif direct sur le cycle de l'eau
}

Marc Mallet ${ }^{1}$, François Dulac ${ }^{2}$, Pierre Nabat ${ }^{3}$, Paola Formenti ${ }^{4}$, Jean Sciare ${ }^{2}$, Greg Roberts 3 , Cyrielle Denjean 4 , Jacques Pelon ${ }^{5}$, Didier Tanré 6 , Frédéric Parol 6 , Gérard Ancellet ${ }^{5}$, Frédéric Auriol 6 , Luc Blarel $^{6}$, Thierry Bourrianne ${ }^{3}$, Gérard Brogniez ${ }^{6}$,

Patrick Chazette ${ }^{2}$, Servanne Chevaillier ${ }^{4}$, Marine Claeys 3 , Aurélie Colomb7, Barbara D’Anna ${ }^{8}$, Yevgeny Derimian', Karine Desboeufs ${ }^{4}$, Jean-François Doussin ${ }^{4}$, Pierre Durand 1 , Anaiis Féron ${ }^{4}$, Hélène Ferré 9 , Laurence Fleury ${ }^{9}$, Evelyn Freney 7 , Philippe Goloub 6 , Noël Grand 4 , Éric Hamonou 2 , Isabelle Jankowiak 6 , Matthieu Jeannot ${ }^{10}$, Dominique Lambert 1 , Jean-François Léon ${ }^{1}$, Sylvain Mailler 11 , Laurent Menut ${ }^{11}$, Géraud Momboisse ${ }^{3}$, José Nicolas 7 , Thierry Podvin 6 , Véronique Pont ${ }^{1}$, Géraldine Rea ${ }^{11}$, Jean-Baptiste Renard ${ }^{10}$, Laurent Roblou ${ }^{1}$, Alfons Schwarzenboeck ${ }^{7}$, Karine Sellegri' ${ }^{7}$, Michaël Sicard ${ }^{12}$, Fabien Solmon ${ }^{13}$, Samuel Somot ${ }^{3}$, Benjamin Torres 6 , Julien Totems ${ }^{1}$, Sylvain Triquet ${ }^{4}$, Nicolas Verdier ${ }^{10}$, Christian Verwaerde 6 , Damien Vignelles ${ }^{10}$

1 Laboratoire d'aérologie, Université Paul-Sabatier / CNRS, Toulouse

2 Laboratoire des sciences de l'environnement et du climat, Institut Pierre-Simon Laplace, CEA / CNRS /

Université Versailles-Saint-Quentin-en-Yvelines, Gif-sur-Yvette

3 Centre national de recherches météorologiques, Météo-France / CNRS, Toulouse

4 Laboratoire interuniversitaire des systèmes atmosphériques, Institut PierreSimon Laplace, Universités Paris-Est Créteil / Paris Diderot / CNRS, Créteil

5 Laboratoire atmosphères, milieux, observations spatiales, Institut PierreSimon Laplace, Université de Versailles Saint-Quentin-en-Yvelines / Université Pierre-et-Marie-Curie / CNRS, Paris

6 Laboratoire d'optique atmosphérique, Université de Lille 1 / CNRS, Lille

7 Laboratoire de météorologie physique, Université Blaise-Pascal / CNRS, Clermont-Ferrand

8 Institut de recherches sur la catalyse et l'environnement de Lyon, Université de Lyon 1 / CNRS, Villeurbanne

9 Service de données de l'Observatoire Midi-Pyrénées, Observatoire Midi-Pyrénées, Toulouse

10 Laboratoire de physique et chimie de l'environnement et de l'espace, Université d'Orléans / CNRS, Orléans

11 Laboratoire de météorologie dynamique, Institut Pierre-Simon Laplace, Université Pierre-et-Marie-Curie / École normale supérieure de Paris / École polytechnique / CNRS, Paris

12 Remote Sensing Laboratory, Universitat Politècnica de Catalunya, Barcelone, Espagne

13 International Centre for Theoretical Physics, Trieste, Italie

marc.mallet@aero.obs-mip.fr

a région méditerranéenne est reconnue comme l'une des régions les plus sensibles aux changements climatiques (Giorgi et Lionello, 2008). En effet, la réponse des modèles climatiques globaux ou régionaux au changement climatique prévoit un assèchement significatif du bassin méditerranéen (diminution de 25-30\% des précipitations, notamment en saison sèche) associé à une hausse importante des températures $\left(+4-5{ }^{\circ} \mathrm{C}\right.$ en été) à l'échéance 2070-2100 pour le scénario A1B. Aujourd'hui, la majorité des études liées au climat présent et futur de cette région ont été effectuées soit à l'aide de modèles climatiques globaux utilisant une résolution horizontale de l'ordre de $150 \mathrm{~km}$, soit à l'aide de modèles dits régionaux (résolution de 
10 à $50 \mathrm{~km}$ ) qui ne prennent pas forcément en compte toutes les composantes du système climatique méditerranéen. La région méditerranéenne est en effet une zone complexe caractérisée par une topographie contrastée, une mer quasi fermée source importante d'humidité, des vents régionaux violents (tramontane, mistral, meltem, bora), ainsi qu'un fort gradient de son albédo de surface entre le sud (désert, semi-aride) et le nord (mer, végétation) du bassin.

Cette région est aussi caractérisée par la présence de différents types de particules atmosphériques, qui peuvent être d'origine anthropique ou naturelle. La mer, les feux de forêts, mais aussi les déserts nord-africains sont des sources importantes d'aérosols. Enfin, l'ensoleillement fort sur cette région associé à des précipitations faibles (notamment lors de la saison « sèche » de juin à septembre) favorise la concentration en particules dites secondaires formées à partir de précurseurs gazeux. Cette région est donc caractérisée par des charges importantes en particules de nature physico-chimique et optique très variée, associées à une forte hétérogénéité spatiale et temporelle. Or, depuis l'expérience Indoex dans l'océan Indien (Ramanathan et al., 2001), on sait que, de par leurs interactions avec le rayonnement solaire et infrarouge, les particules modifient significativement le bilan radiatif à l'échelle régionale, avec des impacts potentiels sur l'évaporation à la surface, les nuages, la dynamique et le climat régional. Ces impacts restent encore à être quantifiés en Méditerranée.

En région méditerranéenne, les divers processus de génération des particules, qui sont soit primaires (c'est-à-dire émises directement sous forme particulaire), soit secondaires, conduisent à une granulométrie et une composition chimique des particules très contrastées. Or, ce sont ces mêmes propriétés microphysiques et chimiques qui contrôlent leurs propriétés optiques (diffusion et absorption) et donc la manière dont les particules interagissent avec le rayonnement (effet radiatif « direct »). Certaines particules comme

1. Mesure le déséquilibre radiatif au sommet de l'atmosphère induit par une perturbation climatique avant que le système climatique n'ait le temps de répondre.

2. Chemistry-aerosol Mediterranean experiment. 3. Mediterranean integrated studies at regional and local scales. les aérosols sulfatés et les sels marins ne font que diffuser la lumière solaire, alors que d'autres comme certaines particules issues de la pollution anthropique ou des feux de forêts, qui contiennent des concentrations élevées en carbone suie, peuvent aussi en absorber une grande partie. Les poussières désertiques, quant à elles, suivant leur composition chimique (présence d'oxyde de fer, notamment) et leur taille, peuvent également absorber une partie du rayonnement visible. Cette absorption est ensuite « redistribuée » sous forme d'échauffement dans les couches atmosphériques où sont localisées les particules absorbantes, modifiant ainsi les profils d'humidité, de température, voire de nébulosité (effet " semi-direct »). La structure verticale des aérosols est donc un paramètre important pour estimer le forçage radiatif ${ }^{1}$ exercé par les particules. Il faut également noter que, de par leur taille (plus importante), les aérosols désertiques ont la capacité d'interagir avec le rayonnement thermique infrarouge. Pour finir, l'albédo de la surface joue également un rôle essentiel. En effet, localisés au-dessus d'une surface réfléchissante, les aérosols absorbants peuvent induire un échauffement au sommet de l'atmosphère. À l'inverse, si les particules sont transportées au-dessus de surfaces faiblement réfléchissantes, comme la surface de l'océan, elles induisent généralement un forçage radiatif négatif (effet refroidissant).

En résumé, toutes ces caractéristiques, à savoir une grande variété de particules, associée à des propriétés physicochimiques et optiques variées, une forte variabilité spatiale et temporelle des concentrations, des interactions avec le rayonnement solaire et infrarouge, un fort gradient sud-nord du type de surface, ainsi bien sûr que le rôle important des interactions océan-atmosphère font du bassin méditerranéen une zone privilégiée pour étudier les interactions entre particules atmosphériques, rayonnement et climat à l'échelle régionale.

\section{Le programme Charmex}

Le but du programme Charmex ${ }^{2}$ (la composante " chimie atmosphérique » du programme international Mistrals ${ }^{3}$ ) et plus spécifiquement de son groupe de travail « aérosol-rayonnement » était d'aborder la question scientifique des interactions entre aérosols, rayonnement et climat sur cette région. L'objectif était d'étudier les possibles réponses de la dynamique atmosphérique, des échanges air-mer, de l'évaporation, de la nébulosité, des précipitations et plus largement du cycle de l'eau au forçage radiatif exercé par les particules. Il s'agit là en effet de questions majeures pour cette région qui connaît une demande en eau toujours plus importante, notamment dans le Maghreb et à l'est du bassin, où aura lieu l'essentiel de la croissance démographique du bassin méditerranéen durant ce siècle. Il apparaît donc primordial d'améliorer notre connaissance de ces processus « aérosol-rayonnementclimat » sur cette région afin de mieux les prendre en compte dans les simulations climatiques futures et de mieux prévoir et anticiper les ressources en eau disponibles.

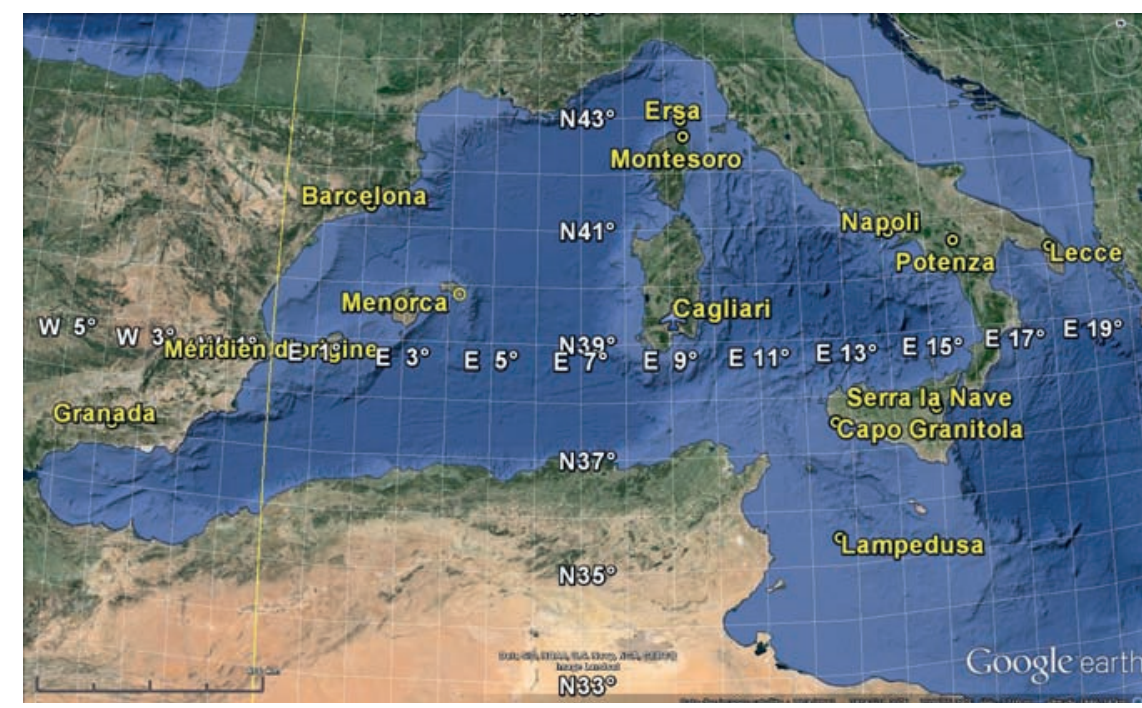

Figure 1. Dispositif instrumental déployé pendant la campagne Charmex/Adrimed (Aerosol direct radiative impact in the Mediterranean) (SOP-1a) sur l'ouest du bassin méditerranéen, avec les différents sites de mesures au sol, ainsi que la base aéroportée de Cagliari. 


\section{Le dispositif expérimental de la SOP-1a}

Un important dispositif expérimental a ainsi été mis en place pendant l'été 2013 (du 10 juin au 5 juillet) sur le bassin occidental de la Méditerranée (figure 1). Cette grande campagne d'observations (SOP-1 $\mathrm{a}^{4}$ ) a permis d'effectuer des mesures in situ sur différentes stations au sol, associées à des observations aéroportées à l'aide des deux avions de recherche français (ATR-42 et F-20 gérés par Safire ${ }^{5}$ ). Des mesures ont également été effectuées à l'aide de ballons sondes et de ballons plafonnants (appelés BPCL, pour ballons pressurisés de couche limite) mis en œuvre par le Cnes. Tous ces moyens expérimentaux ont fourni un jeu de données unique permettant d'étudier les propriétés des aérosols et leurs interactions avec le rayonnement, et d'évaluer la capacité des modèles de climat à reproduire le cycle des particules dans cette région - leur émission, leur transport, l'évolution de leurs propriétés physico-chimiques et optiques, leur structure verticale - dans le but d'estimer leur impact sur le bilan radiatif et le climat.

\section{Les super-sites à l'action}

Dans le cadre de la SOP-1a, deux « super-sites » ont été instrumentés afin de caractériser les propriétés des particules à Ersa, au nord du cap Corse, et sur l'île de Lampedusa (figures 1 et 2). Autant que possible, ces deux sites ont été instrumentés de manière quasi similaire. Deux containers (appartenant au CNRM et au LSCE) ont été mis en place sur le site du cap Corse, et la plate-forme mobile Pegasus ${ }^{6}$ (du Lisa) est venue compléter le dispositif instrumental de la station pour l'observation du climat de l'Enea $^{7}$ sur le site de Lampedusa (figure 2). En parallèle, plusieurs sites secondaires (moins instrumentés que les deux précédents) ont également été utilisés, comme les sites Earlinet-Aeronet de Barcelone, Grenade (Espagne) ou Lecce (Italie). Une nouvelle station a également été déployée sur l'île de Minorque qui a aussi accueilli la station de lancement des ballons. Tous ces sites étaient répartis de façon à pouvoir échantillonner les grands types de particules observés en Méditerranée. Le site d'Ersa était plus
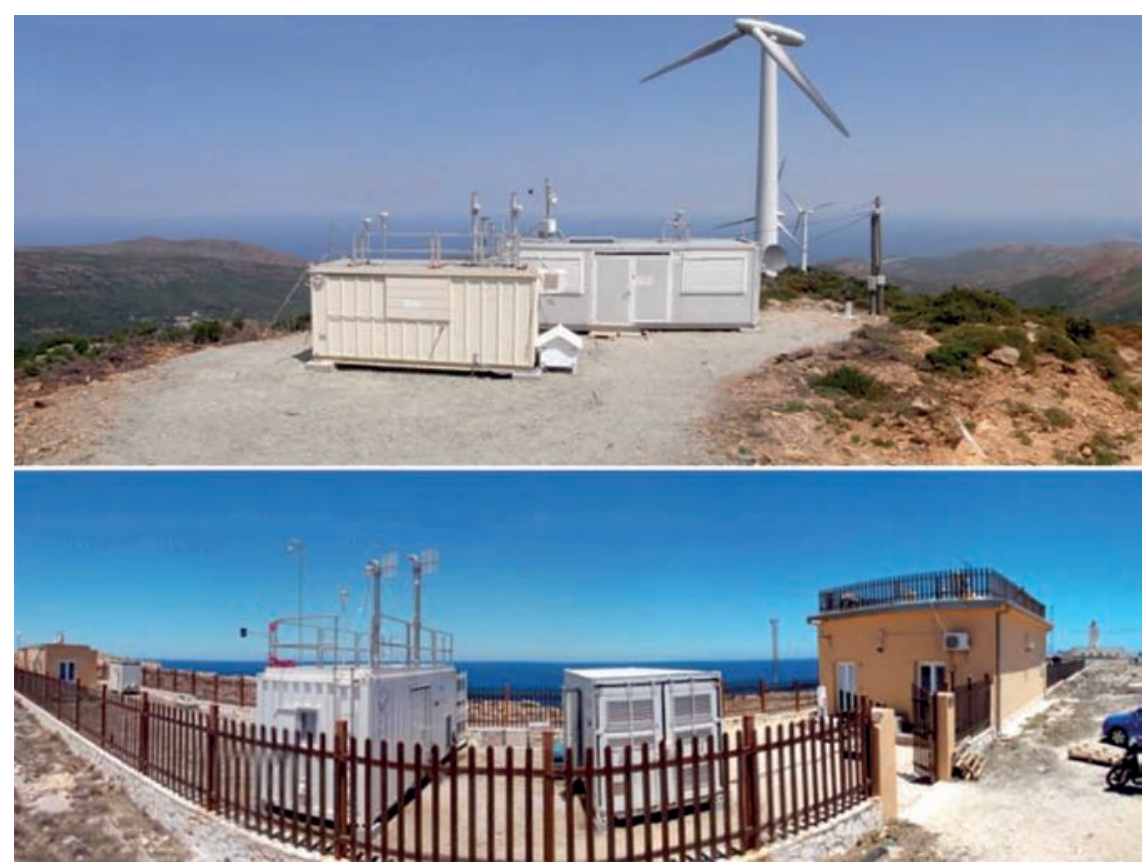

Figure 2. Photo des stations instrumentées du cap Corse (Ersa, en haut) et de Lampedusa (en bas) pendant la SOP-1a.

spécifiquement destiné à l'observation des masses d'air polluées provenant du continent européen (notamment de la plaine du Pô et de la région de Marseille/Berre), alors que le site de Lampedusa était dédié majoritairement à l'observation des poussières désertiques et de la pollution « âgée ». Les sites de Minorque, Grenade et de Barcelone, bien que moins instrumentés, étaient complémentaires, car susceptibles d'échantillonner la pollution depuis la péninsule Ibérique et les poussières désertiques remontant par la mer d'Alboran. Les deux avions de recherche étaient basés à Cagliari (Sardaigne), au centre de la région d'étude, et la localisation de la station des ballons à Minorque avait été choisie pour pouvoir documenter les transports de poussières sahariennes. Les moyens aéroportés étaient déployés en fonction des observations et des prévisions de divers modèles météorologiques et de chimie-transport opérationnels, les produits graphiques étant rassemblés sur un site internet de campagne (http://choc.sedoo.fr). Celui-ci était particulièrement intéressant pour définir les plans de vol des deux avions pendant la campagne.

\section{Les propriétés des particules d'aérosols mesurées à la surface}

Afin de caractériser la granulométrie de l'aérosol sur l'ensemble de son spectre dimensionnel, des compteurs optiques (OPC, optical particle counter) et des instruments à mobilité électrique
(SMPS, scanning mobility particle sizer) ont été utilisés aux stations de surface. En parallèle, des mesures de la composition chimique en espèces solubles (sulfates, nitrates, chlorure...) à haute fréquence temporelle ont également été réalisées à l'aide de systèmes PILS (particle into liquid sampler) aux deux super-sites. Le mélange des aérosols submicroniques a été caractérisé à l'aide d'un V-H-TDMA (volatilizationhumidification tandem differential mobility analyser) sur le site d'Ersa. En parallèle, la composition chimique de la fraction non réfractaire des particules fines (PM1) a également été mesurée à Ersa par un spectromètre de masse et à Lampedusa à l'aide de l'instrument national C-ToF-AMS (compact-timeof-flight aerosol mass spectrometer). Afin de compléter ces mesures en ligne, la composition chimique des particules a également été obtenue grâce à des collectes sur filtres pour différentes gammes de taille.

Les propriétés optiques des particules (diffusion, absorption, extinction ${ }^{8}$ ), très importantes pour notre étude, ont été estimées à l'aide de néphélomètres pour quantifier la diffusion de la lumière dans trois longueurs d'onde. L'absorption du rayonnement par les

4. Special Observation Period.

5. Service des avions français instrumentés pour la recherche en environnement.

6. Portable gas and aerosol sampling units.

7. Agenzia nazionale per le nuove tecnologie, l'energia e lo Sviluppo economico sostenibile.

8. L'extinction correspond à la somme de la diffusion et de l'absorption. 
particules a été estimée à l'aide d'aethalomètres. Cette dernière propriété est cruciale et doit être prise en compte de manière rigoureuse dans les modèles climatiques (Solmon et al., 2008). Elle est souvent représentée par l'albédo de simple diffusion (rapport entre la diffusion et l'extinction du rayonnement). Enfin, des mesures de flux descendants (direct et diffus) dans le spectre visible et proche infrarouge ont été effectuées sur les deux stations. L'instrumentation complète utilisée pour la campagne est détaillée dans Mallet et al. (2015).

\section{Les mesures de télédétection depuis la surface}

En plus des mesures in situ effectuées dans les stations au sol, des observations complémentaires permettant de fournir des informations sur toute la colonne atmosphérique ont été réalisées par télédétection sur les différents sites mentionnés. Principalement, deux types d'instruments ont été utilisés : des systèmes lidars et des photomètres solaires. Les premiers sont des systèmes de télédétection dite active (car munis d'une source de rayonnement laser) fournissant une information sur la répartition verticale des aérosols. Certains systèmes permettent d'obtenir une information supplémentaire sur le type d'aérosols grâce à la mesure de la dépolarisation du rayonnement (due aux particules non sphériques). Ces instruments étaient déployés en continu sur les sites d'Ersa, de Lampedusa et de Minorque, ainsi qu'à Barcelone et Grenade.

En parallèle, des observations effectuées à l'aide de photomètres solaires (à plusieurs longueurs d'onde) ont été obtenues en partenariat avec le réseau Aeronet/Photons (Aerosol robotic network/Photons). Ces instruments de télédétection fournissent des informations sur les particules intégrées sur la colonne atmosphérique. L'un des paramètres optiques dérivés est l'épaisseur optique qui représente l'atténuation du rayonnement sur toute la colonne atmosphérique. Durant la SOP-1a, plusieurs photomètres supplémentaires ont été déployés, en particulier sur l'île de Minorque et à Cagliari. Au total, plus de dix photomètres étaient déployés sur le bassin occidental. Les

9. Observing system including polarisation in the solar infrared spectrum.

10. Photomètre léger aéroporté pour la surveillance des masses d'air.

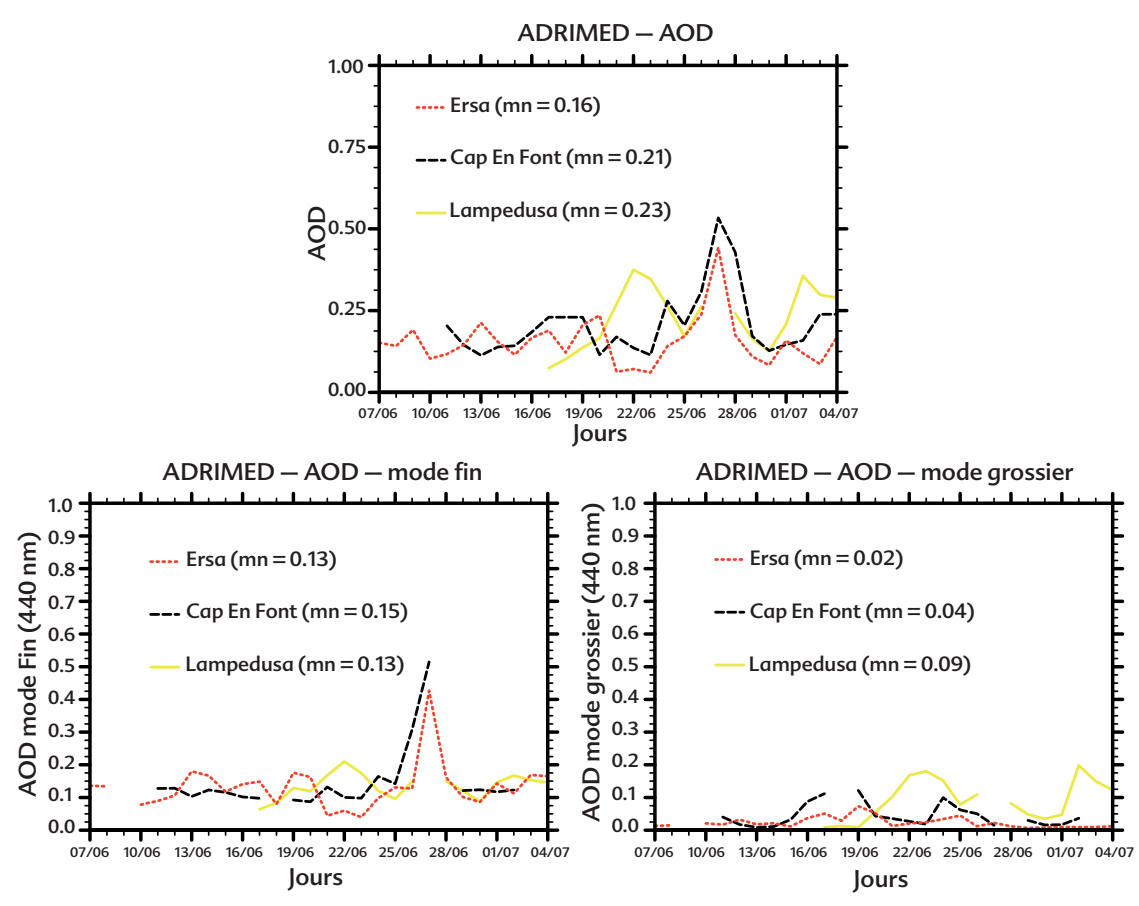

Figure 3. Épaisseurs optiques moyennes journalières (mesurées à $440 \mathrm{~nm}$ ) totale, du mode fin (diamètre de l'aérosol inférieur à $1 \mu \mathrm{m}$ ) et grossier (diamètre supérieur à $1 \mu \mathrm{m}$ ) de l'aérosol, estimées sur les trois stations d'Ersa, de Cap d'En Font et de Lampedusa pendant la campagne SOP-1a. Les moyennes sur toute la période sont indiquées.

mesures d'épaisseur optique à $440 \mathrm{~nm}$ (totale et pour les modes granulométriques fin et grossier de l'aérosol) obtenues sur quatre stations sont rassemblées sur la figure 3 .

\section{Les observations aéroportées (avions et ballons)}

Pour compléter les mesures aux stations de surface, deux avions de recherche, l'ATR-42 et le F-20, ont été utilisés durant la campagne. Ces deux avions étaient basés à Cagliari afin de pouvoir effectuer un survol des deux stations en fonction des conditions météorologiques et du transport des particules. Les mesures in situ des propriétés des particules étaient effectuées à bord de l'ATR-42, celles par télédétection active (LNG, lidar nouvelle génération) et passive (radiomètre Osiris ${ }^{9}$ ) à bord du F-20. Les instruments installés à bord de l'ATR-42 étaient quasi identiques à ceux utilisés à la surface. Cependant, deux instruments récents (SP2, single particle soot photometer, et CAPS, cavity attenuated phase shift) ont été déployés pour la première fois à bord de 1'ATR-42 afin de caractériser la concentration en carbone suie, ainsi que l'extinction du rayonnement dans le visible, apportant une contrainte forte pour l'estimation des propriétés d'absorption des particules. En parallèle, des mesures du radiomètre Plasma ${ }^{10}$ ont également été effectuées dans plusieurs bandes spectrales afin de déterminer la structure verticale de l'épaisseur optique. Enfin, des mesures de flux (descendants et montants) dans les spectres solaire et infrarouge ont été réalisées à bord des deux avions de recherche.

Trois grandes zones du bassin occidental ont été couvertes durant la campagne d'observations SOP-1a en lien avec les conditions synoptiques (figure 4). Lors de la première période (du 17 au 20 juin), les vols ont été principalement effectués sur l'ouest de la zone, incluant un survol et des mesures à plusieurs altitudes (profils verticaux) au-dessus des sites instrumentés de Grenade (figure 5) et de Minorque. Dans un second temps, plusieurs vols ont été effectués sur la zone aérienne militaire française (dénommée D54) permettant d'effectuer des profils au-dessus de la station instrumentée d'Ersa. Enfin, lors de la dernière partie de la campagne, de nombreux vols ont été réalisés sur la zone « Sicile-Lampedusa ». Ces mesures ont permis de caractériser les propriétés microphysiques et optiques des poussières désertiques provenant $\mathrm{du}$ nord de l'Afrique.

Associées aux mesures aéroportées, des observations ont également été effectuées sur des ballons plafonnants et par radiosondages, principalement pour caractériser la granulométrie des particules à l'aide du compteur granulométrique LOAC (light optical 

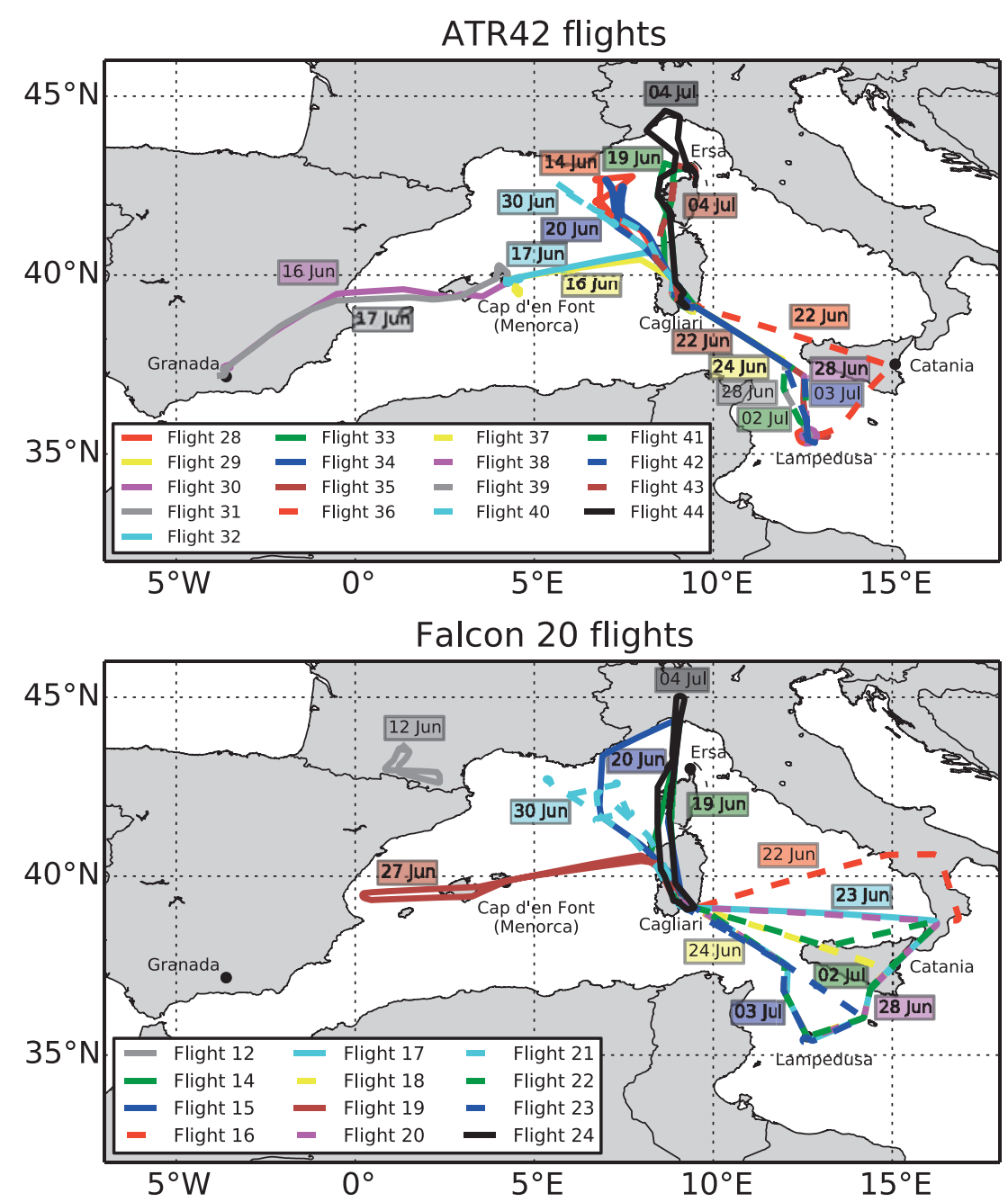

Figure 4. Plans de vols des deux avions de recherche ATR-42 et F-20 effectués lors de la campagne SOP-1a, en juin et juillet 2013.

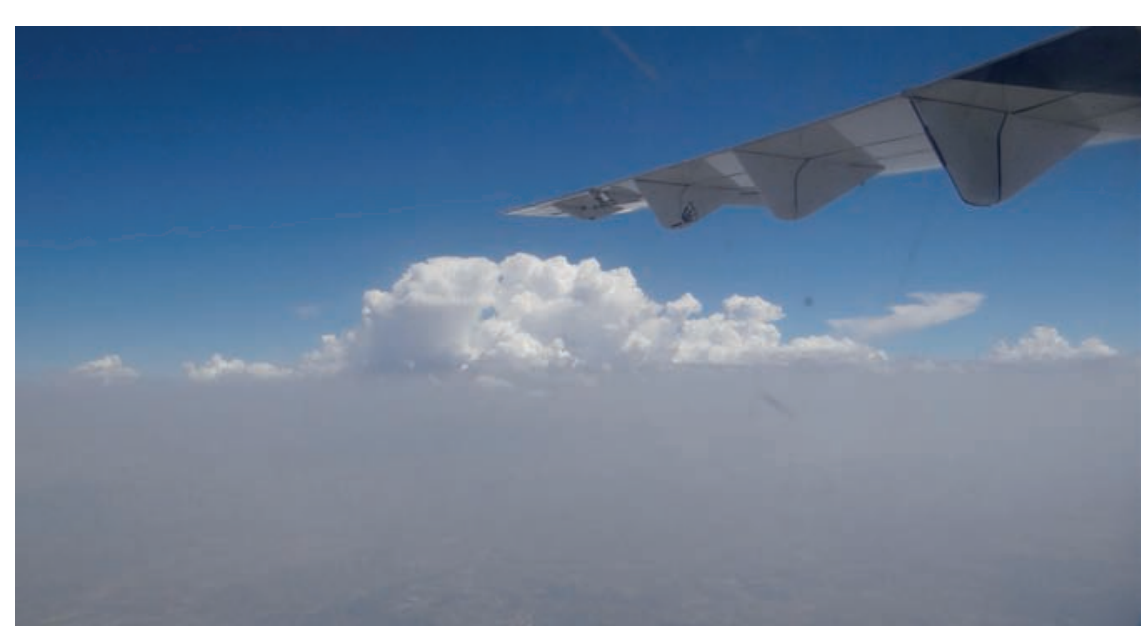

Figure 5. Photo prise à bord de l'ATR42 lors du vol entre Minorque et la station de Grenade où l'on observe très clairement la couche de poussières désertiques.

aerosol counter) développé pour l'occasion, certains ballons embarquant une sonde ozone. Les ballons plafonnants de couche limite sont conçus pour dériver avec le vent à un niveau de densité atmosphérique donné dans la basse troposphère. Quatorze vols de ballons ont été effectués avec succès, entre environ 1850 et $3350 \mathrm{~m}$ d'altitude. Ces ballons ont été lancés sur alertes, notamment lors de remontée de poussières africaines (épisodes du 15 au 19 juin 2013 et du 2 juillet 2013), ainsi que lors du passage d'un panache de feux de forêts d'origine nord-américaine (27-28 juin).

\section{Les conditions météorologiques pendant la campagne}

Les conditions synoptiques rencontrées lors de la SOP-1a ont permis l'émission et le transport de particules désertiques de manière régulière sur le bassin méditerranéen et plusieurs cas ont donc pu être observés et échantillonnés. La figure 6 illustre l'épaisseur optique moyenne quotidienne (à $550 \mathrm{~nm}$ ) obtenue par l'instrument Seviri du satellite $M S G$ sur plusieurs jours pour la période du 16 juin au 2 juillet. Ces observations spatiales indiquent clairement des valeurs modérées de l'épaisseur optique comprises entre 0,1 et 0,6 .

En tout début de campagne, après des pluies dans la nuit du 9 au 10 juin, un flux d'ouest-nord-ouest a régné sur la majeure partie du bassin occidental. Un faible géopotentiel (à $700 \mathrm{hPa}$ ) localisé au large du golfe de Gascogne a progressivement généré un flux de secteur sud-ouest restant faible sur l'Espagne, créant ainsi des conditions favorables à un transport de poussières désertiques gagnant la mer d'Alboran à partir du 12 juin, puis le sud de l'Espagne à partir du 14 juin et les îles Baléares à partir du 16. Ce système dépressionnaire s'est ensuite déplacé sur le golfe de Gascogne à compter du 18 juin, renforçant les vents de secteur sud et entraînant de fortes épaisseurs optiques entre les îles Baléares et la Corse (figure 6).

Après le 20 juin, un régime météorologique différent s'est installé sur le nordouest du bassin induisant un flux majoritairement d'ouest associé à une diminution des charges en particules entre le 21 et le 23 juin sur l'ouest du bassin. Le panache de poussières désertiques se décalant vers l'ouest a entraîné une augmentation de la turbidité sur le bassin central, notamment au niveau de la station de Lampedusa. À partir du 24 juin, un flux de nord-ouest a amené sur le bassin occidental des masses d'air d'origine nord-américaine, avec des épaisseurs optiques fortes du 26 au 28. Enfin, le géopotentiel (à $700 \mathrm{hPa}$ ) observé le 29 juin indique un maximum sur le proche-atlantique induisant un vent de direction nord à nord-ouest sur la majorité du bassin occidental, associé à de faibles valeurs de l'épaisseur optique. Durant la dernière partie de la campagne (30 juin au 5 juillet), des vents de nord à nord-est ont majoritairement transporté des masses d'airs chargées en aérosols désertiques sur le sud du bassin occidental et le site de Lampedusa. 

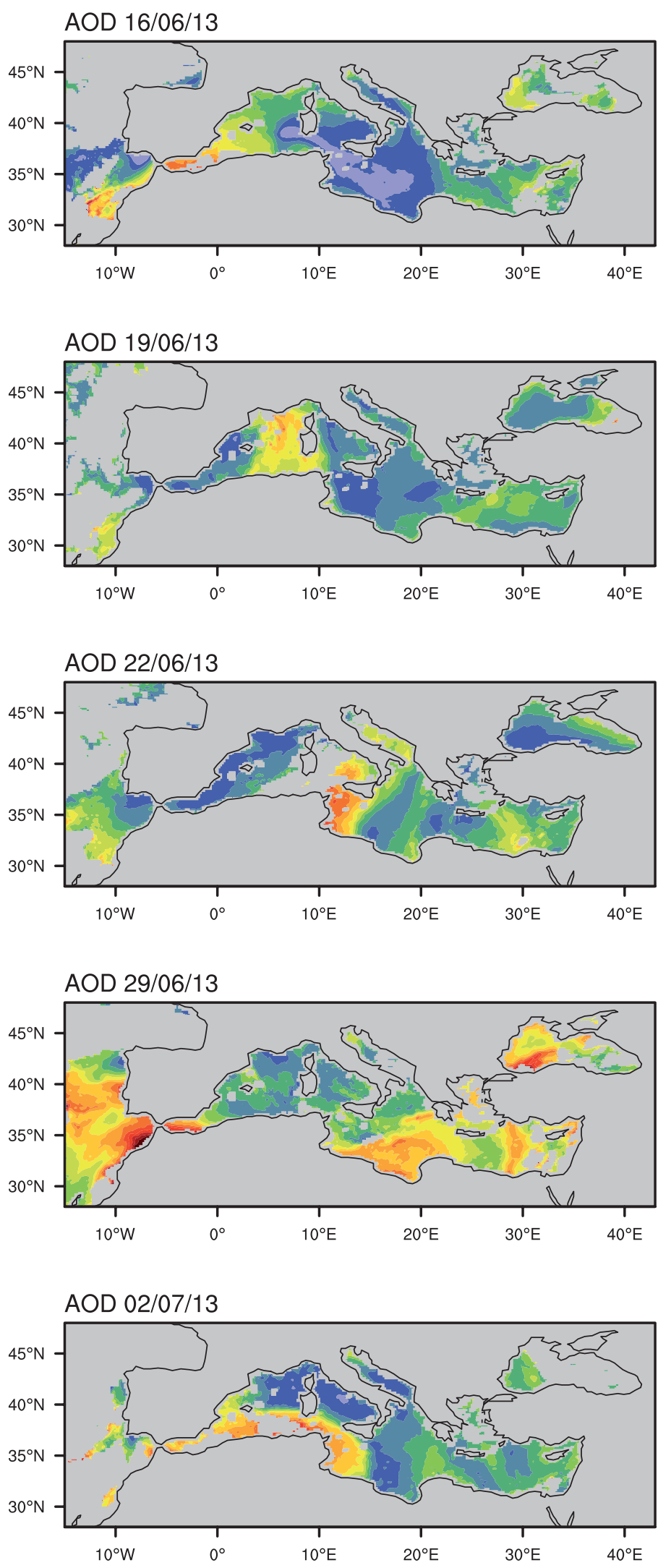

Figure 6. Épaisseur optique en particules ou AOD (aerosol optical depth) restituée à $550 \mathrm{~nm}$ par le capteur Seviri du satellite MSG sur plusieurs journées lors de la période de la campagne SOP-1a. L'échelle de couleur représente I'AOD restituée.

Pour finir, il faut noter que les conditions météorologiques lors de la campagne (absence de températures élevées, périodes de mistral et de tramontane) n’ont pas permis le développement de forte pollution photochimique sur le bassin occidental. Les observations ont donc principalement concerné les poussières sahariennes, des feux de biomasse intense provenant du continent nord-américain autour du 27 juin et les aérosols marins échantillonnés en basses couches lors de périodes de vent modéré.

\section{Résultats issus des observations aéroportées}

La première partie de la campagne a été principalement dédiée à l'étude d'un événement de poussières désertiques observé sur la partie occidentale du bassin entre l'Espagne et les îles Baléares entre le 16 et le 20 juin. Ces masses d'air riches en poussières désertiques ont été particulièrement bien observées par les instruments de télédétection déployées à la surface. Les lidars installés sur l'île de Minorque et au cap Corse ont en effet identifié le passage de ces couches de particules désertiques entre 2 et $4 \mathrm{~km}$ d'altitude à Minorque et entre 4 et $6 \mathrm{~km}$ au cap Corse, où l'on note une augmentation du coefficient d'extinction jusqu'à $0,06 \mathrm{~km}^{-1}$ (figure 7). En parallèle, les données du lidar LNG embarqué à bord du F-20 ont montré une structure verticale complexe avec plusieurs couches de particules bien distinctes au-dessus de la couche limite marine (principalement composée de poussières désertiques). Les sondages utilisant les compteurs optiques embarqués sur l'ATR-42 et sur ballon confirment la présence de particules désertiques jusqu'à plusieurs dizaines de micromètres de diamètre en altitude au-dessus de Minorque du 15 au 19 juin. En parallèle, les observations ont montré peu de variation du diamètre effectif des poussières durant leur transport.

L'utilisation de la télédétection lors de cet épisode montre des épaisseurs optiques modérées (entre 0,1 et 0,7 à 440 et $550 \mathrm{~nm}$, figures 3 et 6 ) avec une contribution équivalente à l'atténuation du rayonnement due aux fractions fine et grossière des particules désertiques (figure 3). Lors de ce premier épisode, des profils verticaux ont été réalisés par 1'ATR-42 au-dessus des sites instrumentés en surface, comme celui du cap Corse. Les propriétés optiques des particules ont donc été déterminées sur la verticale indiquant des coefficients d'extinction pouvant atteindre des valeurs importantes de $100 \mathrm{Mm}^{-1}$ 


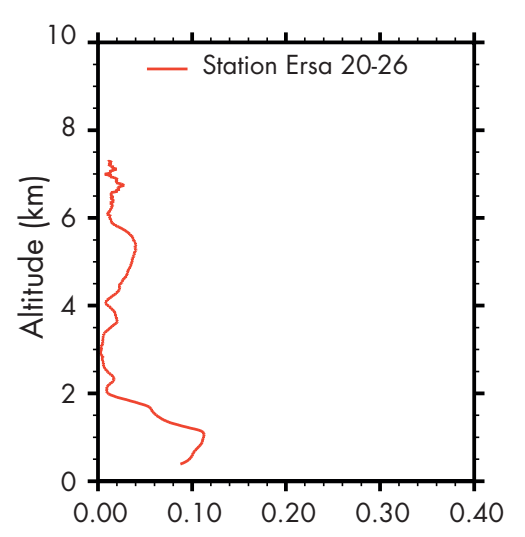

Extinction Particulaire $550 \mathrm{~nm}\left(\mathrm{~km}^{-1}\right)$

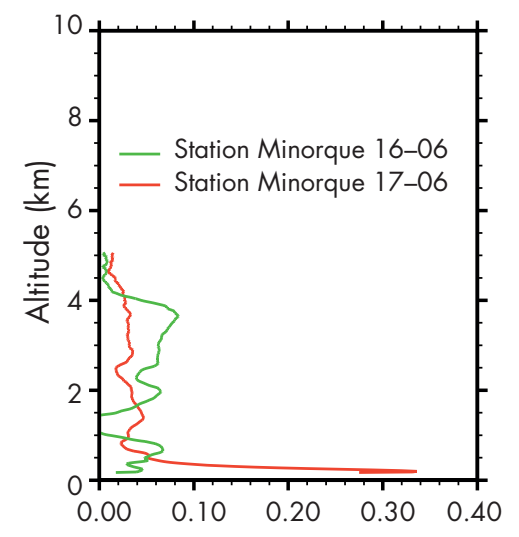

Extinction Particulaire $550 \mathrm{~nm}\left(\mathrm{~km}^{-1}\right)$

Figure 7. Exemples de mesures par lidar (sur les stations d'Ersa et de Minorque) de l'extinction particulaire $\left(\mathrm{km}^{-1}\right)$ à $550 \mathrm{~nm}$ observée entre le 16 et le 20 juin.

$\left(0,1 \mathrm{~km}^{-1}\right)$ pour les couches atmosphériques chargées en poussières minérales. Comme indiqué précédemment, une des originalités de cette campagne a été de coupler des mesures de diffusion et d'extinction afin de contraindre au mieux les propriétés d'absorption des aérosols, qui sont très difficiles à estimer à partir de mesures se fondant uniquement sur la transmission du rayonnement. Cet apport a permis d'estimer de manière plus précise l'albédo de simple diffusion des différents types d'aérosols. Pour ce cas, des valeurs de 0,90 (à $520 \mathrm{~nm}$ ) ont été observées dans les couches de poussières, indiquant un aérosol ayant la capacité d'absorber de manière non négligeable le rayonnement solaire.
Les propriétés optiques mesurées ont ensuite été utilisées dans des modèles de transfert radiatif unidimensionnel afin d'estimer l'impact de ces particules sur le rayonnement solaire à l'échelle locale et à différents niveaux atmosphériques. Les premiers résultats ont indiqué un forçage radiatif direct important à l'échelle locale, avec notamment un impact significatif à la surface lié à une diminution forte du rayonnement solaire due aux particules désertiques. Les premières simulations, validées à l'aide des mesures de rayonnement aéroportées, indiquent en effet une diminution comprise entre 30 et $90 \mathrm{Wm}^{-2}$ à la surface de la mer en fonction de la concentration et des propriétés optiques des particules. Ces valeurs sont tout à fait cohérentes avec certaines déjà référencées sur la Méditerranée par Roger et al. (2006) ou di Sarra et al. (2008).

Le deuxième épisode présenté ici a été observé lors de la deuxième partie de la campagne, qui s'est principalement déroulée sur le bassin central de la Méditerranée. Comme indiqué précédemment, des concentrations importantes en poussières désertiques ont été observées sur la station de Lampedusa le 22 juin, permettant de réaliser des vols aéroportés intéressants sur cette zone en parallèle des mesures effectuées au sol. Les observations réalisées à bord de 1'ATR-42 lors de cet événement indiquent encore une structure verticale complexe (figure 8). Les aérosols désertiques sont principalement localisés en altitude (entre environ 3000 et $6000 \mathrm{~m}$ ) et associés à des maxima de coefficient de diffusion et d'extinction respectivement de l'ordre de 60 et $100 \mathrm{Mm}^{-1}$. Cette couche de poussières est également bien détectée par les mesures de granulométrie avec un mode autour de 8-9 $\mu \mathrm{m}$ caractérisé par de fortes concentrations. Entre la surface et $3000 \mathrm{~m}$, plusieurs couches riches en particules submicroniques sont détectées et notamment une première entre la surface et $1000 \mathrm{~m}$ et une seconde entre 2000 et $3000 \mathrm{~m}$. Dans ce cas, les extinctions observées sont souvent plus faibles que celles détectées dans la couche de poussières.

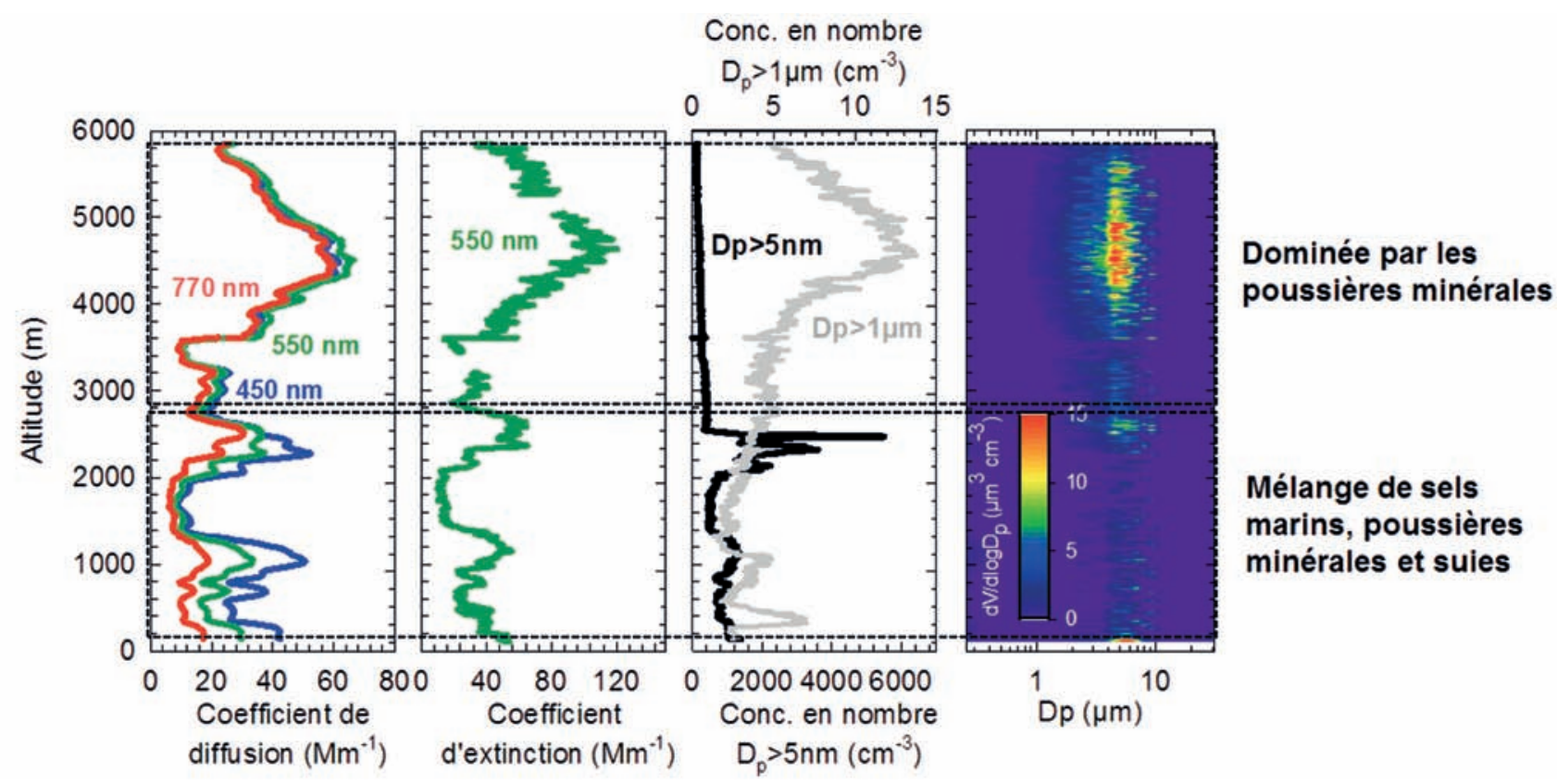

Figure 8. Profil vertical (ATR-42) du coefficient de diffusion, d'extinction, de la concentration en nombre de particules et de la distribution granulométrique en volume, observé au-dessus du super-site de Lampedusa pour le vol du 22 juin ( $D_{\mathrm{p}}$ est le diamètre de l'aérosol). La figure de gauche permet d'observer une forte dépendance spectrale de la diffusion, entre la surface et $3000 \mathrm{~m}$ (aérosol de taille inférieure à 1 um) et une dépendance spectrale plus faible entre 3000 et $6000 \mathrm{~m}$ (aérosol de taille plus importante comme les poussières désertiques). Denjean et al. (2015) 


\section{La modélisation des interactions " aérosols- rayonnement-climat ॥
en Méditerranée}

Quantifier l'impact des particules sur le climat méditerranéen nécessite des modèles météorologiques à haute résolution horizontale (typiquement de 1 à $10 \mathrm{~km}$ ) ou de climat régional (avec une résolution de 10 à $50 \mathrm{~km}$ ), prenant en compte les aérosols atmosphériques et leur influence sur le rayonnement, via un couplage entre le schéma d'aérosol et le code de rayonnement. Généralement, des paires de simulations sont réalisées, en incluant ou non les particules dans les simulations, afin de comparer certaines variables météorologiques (température, humidité relative, couverture nuageuse, précipitations) et de rayonnement entre ces deux simulations. Deux modèles de climat régional sont utilisés dans le cadre de ce projet : le système de modélisation régionale couplée CNRM-RCSM (Sevault et al., 2014 ; Nabat et al., 2015) et le modèle régional de climat RegCM (Giorgi et al., 2012), développé au Centre international de physique théorique (ICTP). CNRM-RCSM est développé au CNRM et intègre notamment le modèle atmosphérique AladinClimat et le modèle océanique Nemomed. Ces deux modèles représentent de manière simplifiée les aérosols atmosphériques, soit en imposant des valeurs mensuelles d'épaisseur optique (fondée sur des données satellites et de modélisation, Nabat et al., 2013), soit en activant un schéma interactif d'aérosols considérant un mélange externe des différentes espèces et utilisant quelques classes de tailles pour représenter le spectre dimensionnel des particules. À l'heure actuelle, ces modèles de climat ne considèrent pas encore les aérosols organiques secondaires (SOA). Cette représentation permet de réaliser des simulations à des échelles «climatiques » (saisonnières, pluri-annuelles et selon des scénarios).

Dans un premier temps, différents modèles ont été évalués sur leur capacité à reproduire l'épaisseur optique en aérosols. La figure 9, montrant des comparaisons entre ces deux modèles de climat (et le modèle de chimietransport Chimere) et les observations Aeronet/Photons sur le site de Lampedusa, indique la bonne capacité des modèles à reproduire 1'ordre de

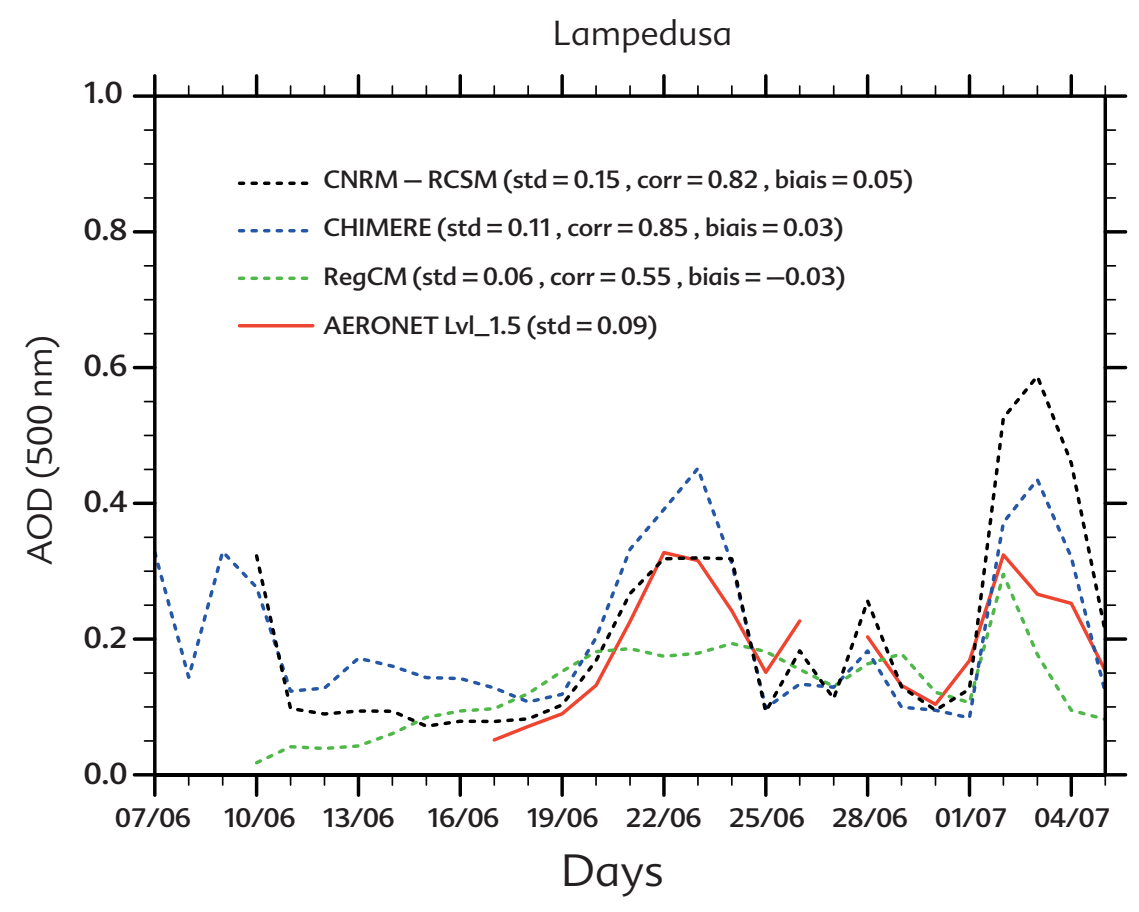

Figure 9. Comparaison entre les simulations de l'épaisseur optique en aérosol (à 500 nm) par les modèles Chimere, CNRM-RCSM et RegCM sur toute la période de la SOP-1a et les observations Aeronet/Photons sur le site de Lampedusa.

grandeur de ce paramètre avec des biais généralement faibles (de l'ordre de $0,05)$. Des tests sont également effectués pour évaluer la capacité des modèles à simuler la granulométrie des aérosols, leur structure verticale et leurs propriétés optiques, à partir des observations in situ obtenues durant la campagne, avant d'effectuer des

simulations sur de plus longues périodes. Des premières simulations de l'effet radiatif direct, effectuées avec les deux modèles de climat régional (figure 10), indiquent des valeurs fortes sur la région euro-méditerranéenne. Les particules induisent en effet une réduction du rayonnement visible à la surface comprise entre 30 et $50 \mathrm{Wm}^{-2}$

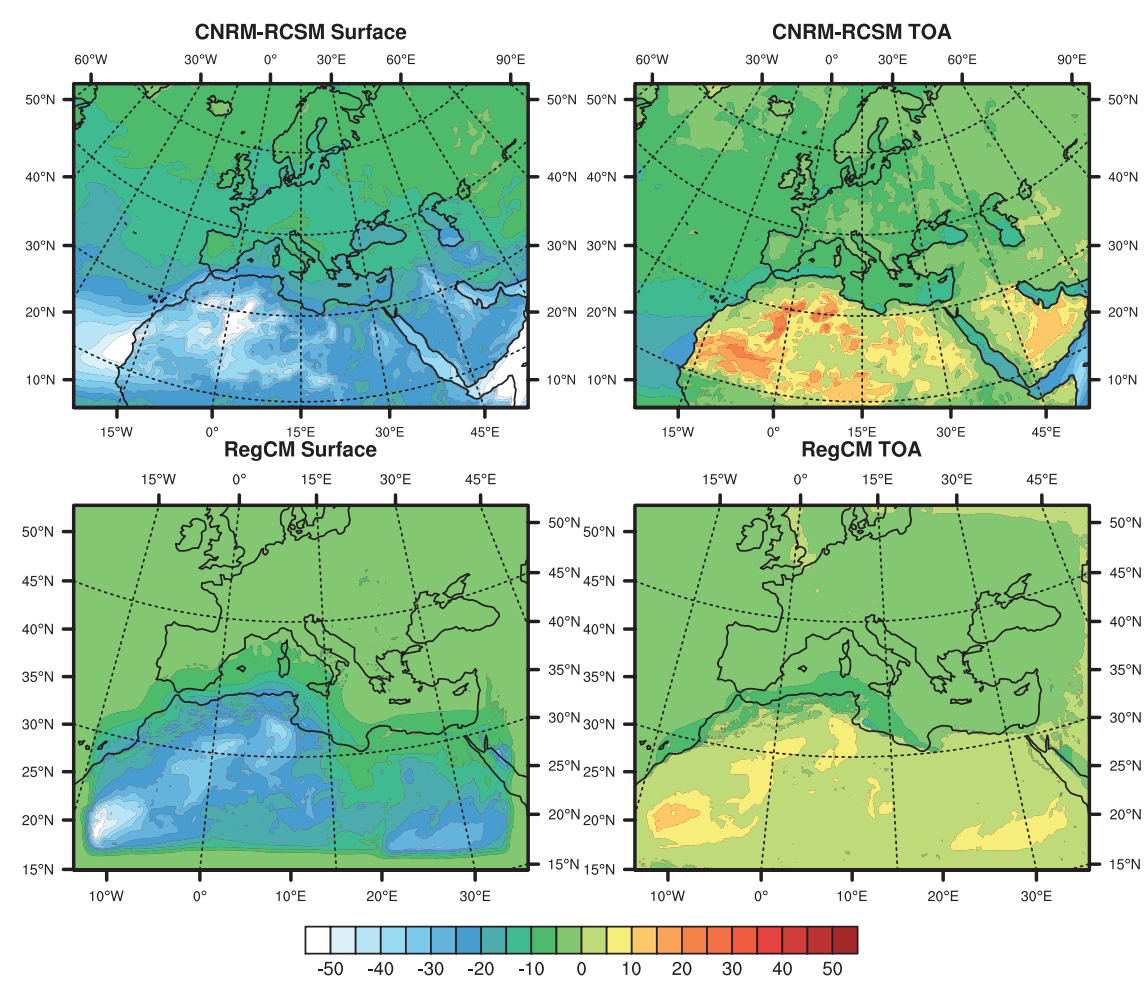

Figure 10. Forçage radiatif direct (pour le spectre solaire uniquement et moyenné sur toute la période de la SOP-1a, en Wm-2) exercé par les aérosols estimé à l'aide des modèles CNRM-RCSM (haut) et RegCM (bas) à la surface (gauche) et au sommet de l'atmosphère (TOA, droite). 


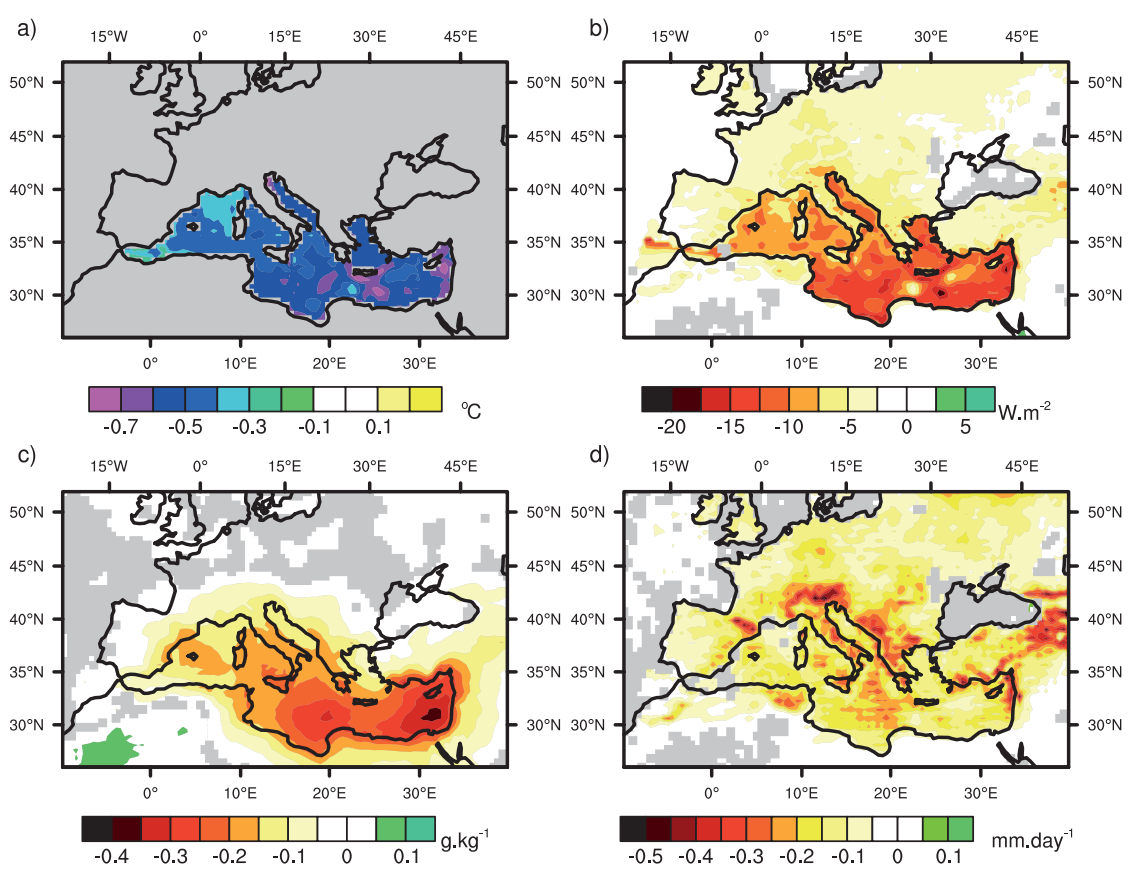

Figure 11. Différence moyenne a) de température de surface de la mer $\left({ }^{\circ} \mathrm{C}\right)$, b) de la perte en chaleur latente $\left.\left(\mathrm{Wm}^{-2}\right), \mathrm{c}\right)$ de l'humidité spécifique $\left(\mathrm{g} \mathrm{kg}^{-1}\right)$ à $925 \mathrm{hPa}$ et $\mathrm{d}$ ) des précipitations (mm jour-1) entre deux simulations incluant ou non les aérosols (naturels et anthropiques). Les moyennes sont réalisées sur la période 2003-2009 et seules les valeurs significatives sont tracées. Adaptée de Nabat et al. (2015).

sur le nord de l'Afrique où sont simulées les plus fortes épaisseurs optiques. Les diminutions sont logiquement plus modérées $\left(\sim 10 \mathrm{Wm}^{-2}\right)$ sur l'Europe et sont principalement liées aux aérosols de pollution sur la zone du Benelux ou des Balkans. Au sommet de l'atmosphère, la figure 10 indique un contraste fort entre le sud et le nord de la région. Sur l'Afrique du Nord, le forçage radiatif est positif, car les aérosols sont localisés au-dessus de surfaces réfléchissantes. À l'inverse, sur la mer Méditerranée et l'Europe, le forçage radiatif direct est négatif, parfois jusqu'à $-15 \mathrm{Wm}^{-2}$.

L'objectif final de ce projet est d'étudier la réponse du climat de la région méditerranéenne à ce forçage radiatif direct et semi-direct. Pour cela, des premières simulations ont été réalisées à des échelles climatiques (simulations pluriannuelles de 2003-2009) à l'aide du système de modélisation régionale couplée CNRM-RCSM, en imposant une climatologie réaliste d'aérosols. Ici, l'une des avancées importantes a été de pouvoir, pour la première fois, estimer l'impact du forçage radiatif des particules sur les flux échangés entre la mer Méditerranée et l'atmosphère, ainsi que sur la température de surface de la mer. Les résultats ont tout d'abord confirmé le forçage radiatif direct important des particules aux échelles climatiques, avec notamment un forçage (dans le solaire) de $-20 \mathrm{Wm}^{-2}$ sur la mer Méditerranée et de $-15 \mathrm{Wm}^{-2}$ sur l'Europe. Le forçage radiatif exercé au sommet de l'atmosphère étant lui de $-10,5 \mathrm{Wm}^{-2}$ (mer Méditerranée) et $-6,5 \mathrm{Wm}^{-2}$ (Europe). Ces simulations ont également indiqué un forçage radiatif semi-direct positif à la surface de $+5 \mathrm{Wm}^{-2}$, dû à la modification de la couverture nuageuse et de la circulation atmosphérique.

Le couplage océan-atmosphère utilisé ici plutôt qu'un forçage climatologique de température de surface de la mer a permis de mettre en évidence l'impact du forçage radiatif des particules sur la température de surface de la mer, avec une diminution de $0,5^{\circ} \mathrm{C}$ en moyenne sur le bassin avec des contrastes marqués (figure 11a). En parallèle, les flux de chaleur latente entre mer et atmosphère sont également fortement modifiés (figure 11b) avec une diminution annuelle moyenne de $11 \mathrm{Wm}^{-2}$ entre les deux simulations incluant ou non les aérosols (naturels et anthropiques), réduisant ainsi l'humidité spécifique dans la basse troposphère de $0,2 \mathrm{~g} \mathrm{~kg}^{-1}$ en moyenne (figure 11c). Les précipitations sont en conséquence aussi réduites par les aérosols sur toute la région méditerranéenne (figure 11d). En moyenne, sur la période 2003-2009, cette réduction atteint $0,2 \mathrm{~mm}$ jour $^{-1}$, soit environ $10 \%$ des précipitations (Nabat et al., 2015).

\section{Conclusions, perspectives}

La campagne Charmex/Adrimed, qui s'est déroulée sur le bassin occidental de la Méditerranée pendant l'été 2013, a été l'occasion de déployer des moyens expérimentaux importants au sol et aéroportés permettant de documenter les propriétés physico-chimiques et optiques des aérosols, leur structure verticale et leur impact sur le rayonnement. Les conditions synoptiques rencontrées lors de la campagne ont engendré des transports réguliers d'aérosols désertiques sur le bassin principalement depuis des régions sources algériennes, marocaines et tunisiennes. Ces mêmes conditions n'ont pas permis le développement d'épisodes de pollution intense pendant cette période. En parallèle, des cas favorables de vents modérés ont permis d'échantillonner des aérosols marins primaires générés à la surface marine.

Ces masses d'air ont conduit à des épaisseurs optiques modérées comprises entre 0,1 et 0,6 (à $440 \mathrm{~nm}$ ) à l'échelle du bassin occidental. Principalement localisées entre 3 et $6 \mathrm{~km}$ et donc non échantillonnées à la surface, les particules désertiques ont pu l'être à l'aide de nombreux vols aéroportées. Au niveau expérimental, l'une des originalités de cette campagne a été l'utilisation de mesures aéroportées d'extinction du rayonnement indépendamment des autres mesures optiques classiquement utilisées. Ces mesures ont révélé des maxima importants $\left(\sim 100 \mathrm{Mm}^{-1}\right.$ à $\left.520 \mathrm{~nm}\right)$ de l'extinction du rayonnement dans certaines couches atmosphériques. L'association des différentes mesures optiques a permis également d'estimer l'albédo de simple diffusion avec une meilleure précision.

Les mesures par lidar réalisées au sol sur plusieurs sites ainsi que celles effectuées à l'aide du lidar LNG embarqué à bord du F-20 ont indiqué une structure verticale complexe avec des couches bien identifiées comme la couche limite marine marquée par des extinctions non négligeables (dues aux aérosols marins et de pollution) associées à de fortes valeurs dans la troposphère libre, typiquement entre 3 et $6 \mathrm{~km}$, liées à la présence des poussières désertiques. Toutes ces informations ont servi d'entrées à des modèles de rayonnement 1-D pour estimer l'impact radiatif direct des aérosols lors du survol des stations. Ces estimations locales ont été complétées de simulations 3-D à l'échelle régionale 
afin d'évaluer l'influence des aérosols sur le bilan radiatif de cette région et ses rétroactions climatiques.

Les premiers résultats issus des simulations régionales ont mis en évidence un forçage radiatif direct important à la surface avec des maxima localisés dans le nord de l'Afrique. Au sommet de l'atmosphère, le forçage radiatif est très contrasté avec des valeurs positives sur l'Afrique du Nord et négatives sur la mer et le continent européen, lié en grande partie aux différences d'albédo de surface. Ces simulations indiquent également des différences fortes entre le forçage radiatif exercé en surface et celui au sommet de l'atmosphère, dues à l'absorption du rayonnement dans les couches atmosphériques où sont localisées les particules. L'impact de ce forçage radiatif direct et semi-direct sur le climat a été étudié ici pour la première fois à des échelles climatiques à l'aide d'un modèle incluant un couplage entre la mer Méditerranée et l'atmosphère. Cet outil de modélisation original (CNRM-RCSM) a permis d'étudier la modification des flux d'humidité, de température de surface de la mer et de dynamique océanique, liée au forçage radiatif des aérosols. Les premières simulations ont indiqué un impact important sur le cycle hydrologique à l'échelle du bassin en réduisant notamment la nébulosité et les précipitations.

Un résultat majeur est l'impact du forçage radiatif des aérosols sur les précipitations, qui sont diminuées de près de $15 \%$ sur certaines régions d'Afrique du Nord comme la Tunisie. Des diminutions sont aussi notées sur certaines parties de l'Europe comme la Grèce. Ces premiers résultats indiquent clairement que les simulations climatiques régionales prévues sur la Méditerranée, notamment dans le cadre de la seconde phase du projet international MedCordex (www.medcordex.eu), devront prendre en compte les différentes espèces particulaires et leurs interactions avec le rayonnement afin d'améliorer le réalisme des simulations du climat dans cette région.

\section{Remerciements}

Ce projet a été soutenu par le programme Mistrals/Charmex (http://charmex.lsce.ipsl.fr) et par des

\section{Bibliographie}

Denjean C. et al., 2015. Size distribution and optical properties of mineral dust aerosols transported in the western Mediterranean. Atmos. Chem. Phys. Discuss., 15, 21607-21669.

di Sarra A., Pace G., Meloni D., De Silvestri L., Piacentino S., Monteleone F., 2008. Surface shortwave radiative forcing of different aerosol types in the central Mediterranean, Geophys. Res. Lett., 35, L02714. doi:10.1029/2007GL032395

Giorgi F., Lionello P., 2008. Climate change projections for the Mediterranean region. Global Planet. Change, 63 , 90-104.

Giorgi F. et al., 2012. RegCM4: model description and preliminary tests over multiple CORDEX domains. Clim. Res. 52, 7-29.

Nabat P., Somot S., Mallet M., Chiapello I., Morcrette J.J., Solmon F., Szopa S., Dulac F., Collins W., Ghan S. Horowitz L.W., Lamarque J.F., Lee Y.H., Naik V., Nagashima T., Shindell D., Skeie R., 2013. A 4-D climatology (1979-2009) of the monthly tropospheric aerosol optical depth distribution over the Mediterranean region from a comparative evaluation and blending of remote sensing and model products. Atmos. Meas. Tech., 6, 1287-1314.

Nabat P., Somot S., Mallet M., Sevault F., Chiacchio M., Wild M., 2015. Direct and semi-direct aerosol radiative effect on the Mediterranean climate variability using a coupled regional climate system model. Clim. Dyn., 44 1127-1155

Mallet M. et al., 2015. Overview of the chemistry-aerosol Mediterranean experiment/aerosol direct radiative forcing on the Mediterranean climate (Charmex/Adrimed) summer 2013 campaign. Atmos. Phys. Chem. Discuss., 15, 19615-19727.

Ramanathan V. et al., 2001. Indian Ocean experiment: An integrated analysis of the climate forcing and effects of the great Indo-Asian haze. J. Geophys. Res., 106, 28371-28398.

Roger J.-C., Mallet M., Dubuisson P., Cachier H., Vermote E., Dubovik O., Despiau S., 2006. A synergetic approach for estimating the local direct aerosol forcing: Application to an urban zone during the "Experience sur site pour contraindre les modèles de pollution et de transport d'émission (Escompte)" experiment, J. Geophys. Res., 111, D13208. doi: 10.1029/2005JD006361

Sevault F. et al., 2014. A fully-coupled Mediterranean regional climate system model: design and evaluation of the ocean component for the 1980-2012 period. Tellus, 66, 23967.

Solmon F., Mallet M., Elguindi N., Giorgi F., Zakey I., Konaré A., 2008. Dust aerosol impact on regional precipitation over western Africa, mechanisms and sensitivity to absorption properties. Geophys. Res. Lett., 35, L24705. doi: 10.1029/2008GL035900 